\title{
Pricing Pseudo Contingencies on Motion Picture Assets under No Free Lunch with Vanishing Risk
}

\author{
Sulaiman Sani ${ }^{*}$, Sihle Precious Maseko', Qiniso Dlamini ${ }^{1}$, Firdausi Adamu Abdullahi² \\ ${ }^{1}$ Department of Mathematics, University of Eswatini, Kwaluseni Campus, Kwaluseni, Eswatini \\ ${ }^{2}$ Department of Mathematics and Computer Science, UMYU, Katsina, Nigeria \\ Email: *ssulaiman@uniswa.sz, solarsihle@gmail.com, 150975@student.uniswa.sz, firloloh@gmail.com
}

How to cite this paper: Sani, S., Maseko, S.P., Dlamini, Q. and Abdullahi, F.A. (2020) Pricing Pseudo Contingencies on Motion Picture Assets under No Free Lunch with Vanishing Risk. Journal of Mathematical Finance, 10, 525-535.

https://doi.org/10.4236/jmf.2020.104032

Received: August 31, 2020

Accepted: October 11, 2020

Published: October 14, 2020

Copyright $\odot 2020$ by author(s) and Scientific Research Publishing Inc. This work is licensed under the Creative Commons Attribution International License (CC BY 4.0).

http://creativecommons.org/licenses/by/4.0/

\begin{abstract}
Motion picture derivatives have proved valuable in hedging financial risk of the movie industry. However, the existence of pseudo assets within certain category of movie contingencies makes market trading below capacity due to hyper level pricing arbitrage. This paper analyzes pseudo assets lying within movie contingencies and develops a stochastic pricing strategy under no arbitrage condition. Demonstration of the application of derived formulas is provided as examples and remarks over imposed effects sequel to sub pseudo contingencies. Accordingly, the results derived here are aids of assistance for movie investors relative to financing pseudo asset ventures for the purpose of valuation.
\end{abstract}

\section{Keywords}

Motion Pictures, Pseudo Asset, Pricing Strategy, Free Lunch with Vanishing Risk, Contingencies

\section{Introduction}

Motion picture is a collection of film making institutions comprising production, screen writing, festivals, distribution and marketing companies. It is the biggest leisure industry in the universe and generally expensive to run. As a result, institutions within the industry connect with other industries for equipments, securities, marketing and so on. The largest movie markets by box office are the US, China, UK, Japan and India. Countries with largest movie productions are India, Nigeria, US, UK, and France and area with largest movie productions is Hong Kong in China (https://en.wikipedia.org/wiki/Film_industry). Motion pic- 
ture has enjoyed and still is enjoying good market statistics; for instance, Hollywood reporter of 21st March 2019 indicates that worldwide theatrical market has box office worth of 41 billion USD as at 2018. Top 3 regions by box office gross earnings as at 2016 are Asia-Pacific (14 billion USD), North America (11 billion USD) and Europe together with the Middle East and North Africa (11 billion USD).

Hollywood is the name of the US ${ }^{1}$ motion picture. It is the oldest motion picture in history. In 2011, Motion Picture Association of America (MPAA) identifies Hollywood; "a major employer" in the US supporting over 2.2 million jobs worth 137 billion USD in total wages as at 2009. Direct jobs generate 40.5 billion USD in wages with average salary $26 \%$ higher than the national average. Jobs in core business of production, marketing, manufacturing and distribution of films stand at 272,000 [1]. Bollywood is Hindi motion picture with $43 \%$ India's net box office revenues. In the Annual World Economic Forum meeting 2019, [2] identifies Bollywood contents as "globalised" leading to economic growth and markets. Nollywood is the Nigerian motion picture producing films in English, Yoruba, Hausa and Igbo languages. Moudio of the Africa Renewal calls "Nollywood" a potential gold mine in both employment and revenues (https://www.un.org/africarenewal/magazine).

Although, multilingual audiovisual add-ons have been present in movies of any wood as assets and contingencies only recently, mathematicians and other scholars become interested in their forms [3]. The "interested" here implies how surjectivity due to human complexities can be made a tool for business. For instance, surjectivity made French people prefer movies in French to any language [4]. As such, we see complete localization works in French taste for French movie markets. Animated movies like Wreck it Ralph, released as Le Monde de Ralph, with flawless French dubbing leaves one thinking that originally the movie was in French.

The Koreans love not just English but American English. Korean surjection is passionate to Americanised English [5]. Thus, movies for Korean markets are mostly undubbed in Korean. The Arabs love Arabic and English simultaneously [6]. Arab's surjection is having both experiences simultaneously. Consequently, movies for Arab markets have subtitles that keep the original English title or literal translation (Arie Barak) and the Arabic subtitles. Herein Africa, movie customers are obsessed with voice over dubbing movies in the local languages with selected foreign episodes. In the African surjection, episodes and languages can influence movie market and finances. Be it may, global motion picture appear happy to add-on movies through localization to attract market and higher profit. Unfortunately, some pricing misfits exist with future market consequences.

In his 1964 book; "From Understanding Media" [7], describes the media as a powerful means of control since different cultures are represented verbally, visually and orally. This description implies that effective add-on modes as assets ${ }^{1}$ Originated over 121 years ago. The earliest account of film in USA dates back to June 1894 in Richmond, Indiana by Charles Frances Jenkins. 
in movie media can contribute to reception and market. The "converse" suffices to justify why a hit movie production in a relevant market may go burst in another market. The book Nonlinear System Identification: by [8] posits the nonlinearity of languages rather than customary additive belief held in some quotas. In this connection, movies with add-on functions of subtitles, dubbings and voice overs can gain increasing market if embedded add-ons are necessary shifts of nonlinear movie spaces. They can be jewelries of global motion picture.

One way trading add-ons is positive to motion picture is realistic financing. Movie markets in several spots round the globe trade add-ons (dubbings, subtitles, voice over, translations etc.) with little or no market value. Particular case is the sameness in price of the movie tyrant directed by Gideon Raff with or without add-ons. This price misfit holds for most movies of any wood. There are consequences for this mis-pricing act especially on investment capital. On records, add-on processes are not free lunches since resources are drained in the process. Price insensitivity and mis-fits reflecting as zero add-on prices in movies can result only in market arbitrage [9]. As such, limiting market stability is near impossible a.s.

Moreover, investophobia sequel to risk tendencies capable of crunching movie markets with maximum impacts on movie driven economies is within premise. Clearly, there is need to trade add-ons as contingencies for additional profit. Generally, asset pricing are settled through trading in financial markets where prices of underlying assets sometimes tend to be volatile especially for unawared assets such as pseudo assets and derivatives portfolio. The eventual consequences like market inefficiency present severe negative implications on motion pictures [10].

Here, arbitrageurs manipulate the market to gain excess returns while real investors lost more than expected leading to deadweight loss [11]. Price informativeness often affects decision making in what is known as the feedback effect [12]. From an economic standpoint, unlawfulness is a disadvantage that repels future capital flow as investors prefer stable markets to unstable ones. Moreover, underpricing of pseudo movie assets has dire consequences on businesses including loss of profits, unemployment and eventual loss of investment resources. Underpricing of assets is tricky as a long-term strategy in businesses in its inability to attain good profit margin since it requires constant ongoing and stream of high volume sales. The performance of businesses is contingent to its pricing strategy, therefore it is crucial to develop an efficient pricing extension for pseudo assets to ensure business attainability.

\section{Literature Review}

Add-oning is Herculean in the least so to speak. Darbeltnet's model presented in [13] divides translation strategies into direct and oblique ${ }^{2}$. Take the case of

${ }^{2}$ Direct translation consists of borrowing, calque, and literal translation whereas oblique translation consists of transposition, modulation, adaptation and equivalence. 
add-oning a post war movie by functional equivalence. Though, functional equivalence trivially can differentiate language words, the star translator must decide which translates are known to general movie public for better market. The language system and the difference between source and target cultures [14] can make not just the enforcement of literal translations impossible but the need for caution for market reasons. In [15], movie voice is proposed to be distinct from artist's voice and movie tones be made monotonically continuous. This proposal makes add-oning above earth since time between semantic and style (difficult control procedures) can bounce a supposed-be hit movie. [16]'s example of semantic and stylistic losses on the Danish TV series Charlot and Charlotte for voice-over in Lithuanian suffices in this respect.

[3]'s message-body movements matching compounds the task of add-oning to the next level since voice isochrony requires translation-fitting. Though, [17]'s suggestion on invisibility and obtrusiveness principles helps in this regard, the voice artist must deliver the text speech systematically to reduce overall effects. Besides, such manipulations under less adequate care create viewer illusions capable of bouncing a good movie in gross earnings. While in some quotas, movie bosses may argue that pricing add-ons will affect the art involved in producing great movies; this position does not financially add value to global motion picture in the least. As in the wall street journal of 29-30 April 2006, the idea of investing in motion picture has attracted hedge funds and money managers to the industry. Consequently, the future holds good for add-on commercialization since add-on prices increase profit margins a.s.

[18]'s coupling marketing actions and movie quality on box office performance is good for add-on pricing extensions. Again, [19]'s distribution forecasts and box office performance creates forecasting methodology for add-ons. Designed flow model with target subgroups makes it awesome to incorporate "at all cost" to carve a notch for add-on prices. [20]'s separable dependence of box office performance just after release could be re-modeled to include "add-ons with non-zero prices". Additional Data field of 1956 movies from 1985 to 1999 be extended in the neighborhood of year 2019 movie spectrum and the assumed consumer's utility to watch a movie incorporates "given $n \in \mathbf{N}$-size add-ons with price $p \in \mathbf{R}$ ". More specifically, the utility consumer $i$ receives from movie $j$ on week $t$ be transformed to somewhat

$$
u_{i j t}=\theta_{j}\left(\chi_{n}\right)-\lambda\left(t-r_{j}\right)+\xi_{j t}+\zeta_{i t}+(1-\sigma) \epsilon_{i j t}
$$

where $\theta_{j}$ is quality of movie $j$ under add-on prices, $t-r_{j}$ is the elapsed time in weeks since release, $\xi_{j t}$ is a week's random effect. With this extension, the individual error term $\zeta_{i t}+(1-\sigma) \epsilon_{i j t}$ can be transformed by suitable choice of pricing functions enough to include errors from add-on prices so that Einav's share for movie $j$ in week $t$ logit is

$$
\log \frac{S_{j} t}{S_{0} t}=\theta_{j}\left(\chi_{n}\right)+\tau_{t}-\lambda\left(t-r_{j}\right)+\sigma \log \left(\frac{S_{j} t}{1-S_{0} t}\right)+\hat{\epsilon}_{j} t
$$


Studios for production, distribution and marketing add-ons may follow [21]'s model. Basic assumptions include cost of add-ons $c>0$ embedded within the cost of movie purchase or rentage. Under perfect competition where each consumer has a unit valuation $v>(\epsilon+c) ; \epsilon>0$, vital results could be derived for several distributions $F(\cdot)$. Again, add-on options can be valuated at ease similar to other known stocks in and out of global motion pictures.

[22]'s procedures are ideal for performance analysis of movies with add-ons. Inventory level checks within chosen time frames for the two movie types be evaluated after stocking. Calibrations then can follow any structural econometric model of store-add-on/not add-on level using among others; the Generalized Method of Moments (GMM). [23]'s optimal releasing time is convenient for analyzing how consumer expectations on anticipation times of add-on videos release based on their past experiences for better market. [24]'s model can capture box office gross earnings under add-ons prices. In addition, it can provide requisite fitting-in-analysis given add-on size $n \in \mathbf{N}$.

[25]'s behavioral model could be adopted for market behaviour analysis of movie clients when add-on prices are incorporated within movie price mix. [26]'s predictor for box office performance before release is good for predicting box office performance under money effects of add-ons since, extra parameter addition is present. Thus, it would not be extra terrestrial to include add-ons within the algebra. Nonetheless, distributors could forecast gross box office performance of add-on movies by "bench-marking" against same/similar movies without add-ons based on the added price characteristics.

[27]'s game-theory approach to strategic competition could be adopted in case one movie contains add-ons and the other not. The two movie types could be characterized by marketability parameter $\left(\alpha_{i}, \alpha_{j}\right)$ and playability parameter $\left(\beta_{i}, \beta_{j}\right)$. Revenue in each movie class be captured using any form of the shareattraction model assuming that movie attraction $i(t)$ follows any finite distribution. Under this formulation, the Nash equilibria, limits and solutions could be found using several analytic and numerical techniques [28]. In the area of pricing add-ons, the [29]'s option pricing model is awesome for use sequel to small number of input parameters. In this respect, add-on option valuation could be based on perfect replication of contingent claims when investors replicate add-on options by continuously re-balancing supply under self-financing portfolio, stocks and risk-free add-on bonds assumptions. The price of the add-on option could then be determined by the price of the replicating add-on assets in trade.

[30]'s silver-screener could tackle scheduling problem relating to parallel pricing associated with add-ons in operations research. In this analogy, screens could be modeled as movies while add-ons are jobs. Thus, assigning a particular movie on screen is then that of assigning jobs to machines. This analogy could enable the set up of mathematical programming model for optimal allocation of add-ons to screens. 
[31]'s contract suffices for the law of contract governing add-on prices between manufacturers and retailers. Add-on pricing law of contract is necessary to mitigate possible manufacturer-retailer conflicts. The retailing tail driven by own profit greed may take actions that deviate from those preferred by the manufacturers and eventually may affect profit margins. Again on records, prior to 1998, most videos were sold to retailers at a price about 70 USD. Afterwards, contracts for many movies switched to two-part tariffs with retailers charged a price about 5 USD per video but had to unhappily remit more than $50 \%$ of rental income to the distributor [22].

Though, [21]'s revenue sharing contracts has the capacity to nullify most problems associated to contracts in this sphere, the "unhapiness" provides a strong ground for theoretical development and empirical examination of contract theories for add-ons. Policy wise, [32]'s support system matching between decision makers and decision making is good for add-on prices. The implementation of a decision support system for add-on prices should then depend on add-on manager's willingness to provide better forecasting units otherwise, a forecasting system be developed with minimal managerial involvement. [33]'s retailing numbers for movies with add-ons to be ordered should keep the add-on dynamics fluidy in time, quantity and space for optimal coordination of the add-on business for better motion pictures.

Unfortunately, even with these clear cut fitting demonstrations, a pseudo movie contingency can challenge movie pricing sequel to fears of arbitrage and its financial consequences. This is in view of the hidden un-priced sub assets like add-ons within the majority of movie contingencies. This article fills some gaps by defining pseudo assets and contingencies and proves existence of arbitrage therein pseudo assets of movie spaces and finally, provides pricing strategy for such assets and portfolios.

\section{Basic Assumptions}

We consider a compound ${ }^{3}$ movie asset $A$ in trade given by

$$
A=\sum_{i=0}^{n} A_{i}
$$

where each $A_{i} \in A$ is priced $X_{i}($.$) . Suppose that one investor holds portfolio$ $\theta(A)$ when the price $X(A)=X_{i}($.$) . Then \theta(A)$ is a pseudo portfolio for asset $A$. Evidently, $\theta(A)$ has arbitrage tendency in view of its trading price $X_{i}$ corresponding to that of $A_{i} \in A$. If $\theta(A)$ is admissible then, by [9], $V(t, A)$ exists a.s.

Intuitively, $A$ in (3) is an arbitrage asset and as earlier motivated, there is need to construct an arbitrage free pricing strategy for such movie assets. To prove that any $\theta(A)$ is an arbitrage, suppose that the price $X(t, \omega, A)$ for $A$ is such that

${ }^{3} \mathrm{~A}$ movie with $n>1$ independent components. 


$$
\mathrm{d} X(t, \omega, A)=\mu_{i}(A) X(t, \omega, A) \mathrm{d} t+\sigma_{i}(A) X(t, \omega, A) \mathrm{d} B(t)
$$

where $\mu(A)$ is the drift coefficient of the movie market and $\sigma(A)$ is the volatility coefficient under the white noise $B($.$) . Applying the arbitrage criteria in$ [9] on (4), we have

$$
\left[\begin{array}{ll}
\sigma_{11}(A) & \sigma_{12}(A) \\
\sigma_{21}(A) & \sigma_{22}(A)
\end{array}\right]\left[\begin{array}{l}
U_{1} \\
U_{2}
\end{array}\right]=\left[\begin{array}{l}
\mu_{1}(A) \\
\mu_{2}(A)
\end{array}\right]
$$

So that

$$
\begin{array}{r}
\sigma_{11}(A) U_{1}+\sigma_{12}(A) U_{2}=\mu_{1}(A) \\
\sigma_{21}(A) U_{1}+\sigma_{22}(A) U_{2}=\mu_{2}(A)
\end{array}
$$

In view of (3), (6) and (7) have the representation that

$$
\begin{aligned}
& {\left[\begin{array}{c}
\sigma_{11}\left(A_{1}\right) \\
\sigma_{11}\left(A_{2}\right) \\
\vdots \\
\sigma_{11}\left(A_{n}\right)
\end{array}\right]\left[\begin{array}{c}
U_{1} \\
0 \\
\vdots \\
0
\end{array}\right]+\left[\begin{array}{c}
\sigma_{12}\left(A_{1}\right) \\
\sigma_{12}\left(A_{2}\right) \\
\vdots \\
\sigma_{12}\left(A_{n}\right)
\end{array}\right]\left[\begin{array}{c}
U_{2} \\
0 \\
\vdots \\
0
\end{array}\right]=\left[\begin{array}{c}
\mu_{1}\left(A_{1}\right) \\
\mu_{1}\left(A_{2}\right) \\
\vdots \\
\mu_{1}\left(A_{n}\right)
\end{array}\right]} \\
& {\left[\begin{array}{c}
\sigma_{21}\left(A_{1}\right) \\
\sigma_{21}\left(A_{2}\right) \\
\vdots \\
\sigma_{21}\left(A_{n}\right)
\end{array}\right]\left[\begin{array}{c}
U_{1} \\
0 \\
\vdots \\
0
\end{array}\right]+\left[\begin{array}{c}
\sigma_{22}\left(A_{1}\right) \\
\sigma_{22}\left(A_{2}\right) \\
\vdots \\
\sigma_{22}\left(A_{n}\right)
\end{array}\right]\left[\begin{array}{c}
U_{2} \\
0 \\
\vdots \\
0
\end{array}\right]=\left[\begin{array}{c}
\mu_{2}\left(A_{1}\right) \\
\mu_{2}\left(A_{2}\right) \\
\vdots \\
\mu_{2}\left(A_{n}\right)
\end{array}\right]}
\end{aligned}
$$

Each $\mu_{i}(A)$ depends on $A_{i}$ since each iteration drifts independently of the sub assets. Moreover

$$
\sigma_{1, j}\left(A_{1+k}\right)= \begin{cases}\sigma_{1, j} ; & k=0 \\ 0 ; & k>0\end{cases}
$$

Note that the left hand sides of (8) and (9) are column matrices and multiplication is impossible implying that the $U$ s do not exist and hence $\theta(A)$ is an arbitrage. For convenience, we choose the Ornstein-Uhlenbeck price path for $X(t, \omega, A)$ as a special case of (4) such that

$$
\mathrm{d} X(t, \omega, A)=\mu(A) X(t, \omega, A) \mathrm{d} t+\sigma(A) \mathrm{d} B(t)
$$

and provide arbitrage free pricing strategy for $A$ as motivated in this work.

\section{Results}

Lemma 4.1 The arbitrage free price $p$ for a pseudo-movie asset $A$ with $n$ independent sub-assets $A_{i}$ 's $i=1,2,3, \cdots, n$ is given by

$$
p=X(0, \omega, A) \mathrm{e}^{\mu(A) T}+\int_{0}^{T} \sigma(A) \mathrm{e}^{\mu(A)(T-t)} E[\mathrm{~d} B(t)]
$$

Proof Suppose $p \sim E[X(t, A)]$ and define $f: \mathfrak{A} \rightarrow \mathfrak{R}$ where $\mathfrak{A}$ is the space of assets such that for $A \in \mathfrak{A}$, we have $f(t, A)=f_{t}(A)$ with the condition that for any $A_{i}, A_{j} \in A ; i \neq j$, we have 


$$
f\left(t, A_{i}+A_{j}\right)=f_{t}\left(A_{i}+A_{j}\right)=f_{t}\left(A_{i}\right)+f_{t}\left(A_{j}\right)
$$

Again, for $i=j=k=m$ then

$$
f\left(t, A_{i}+A_{j}+A_{k}+\cdots+A_{m}\right)=f_{t}\left(m A_{i}\right)=m f_{t}\left(A_{i}\right)
$$

Given (11), the integrating factor is $\mathrm{e}^{-\mu(A) t}$ and multiplying the given equation by this factor and upon basic simplification in the interval $[0, T]$ yields

$$
X(T, \omega, A)=X(0, \omega, A) \mathrm{e}^{\mu(A) T}+\sigma(A) \int_{0}^{T} \mathrm{e}^{\mu(A)(T-t)} \mathrm{d} B(t)
$$

with expected price $p$ equals to

$$
p=X(0, \omega, A) \mathrm{e}^{\mu(A) T}+K ; K=0,1,2,3, \cdots
$$

The lemma follows in view of (13) and (14) directly.

Intuitively, the price $p$ in (16) is arbitrage free since each sub price value $X\left(A_{i}\right)$ is taken into account in the final pricing of $A$. We see how this pricing works in the following examples.

Example 4.1 A movie retail shop sells a movie contingency $A$ of three independent sub contingencies: the movie, the voice over and translation that costs $E$ 20, $E 3$ and $E 2$ respectively at a price $E 30$. The retail shop operates from noon to 7 p.m. Show whether the price $p(A)$ has arbitrage ${ }^{4}$.

Solution 4.1 Here, the business trading period $T=\frac{t}{24}=\frac{7}{24}$ since there are 7 hours between trading hours. Again, $X_{0}\left(A_{1}\right)=E 20, X_{0}\left(A_{2}\right)=E 3$ and $X_{0}\left(A_{3}\right)=E 2$. In view of $(16)$, we have

$$
p=X_{0}\left(A_{1}\right) \exp (T)+X_{0}\left(A_{2}\right) \exp (T)+X_{0}\left(A_{3}\right) \exp (T)+K, K=0,1,2,3, \cdots
$$

Upon substituting the values of above in (17), we have

$$
p=E 20 \exp \left(\frac{7}{24}\right)+E 3 \exp \left(\frac{7}{24}\right)+E 2 \exp \left(\frac{7}{24}\right)+K
$$

where $K=E\left[\int_{0}^{T} \exp (T-t) \mathrm{d} B(t)\right]$. So that

$$
p=E 26.774+E 4.016+E 2.677+K=E 33.5 ; K=0
$$

In view of (12), $p(A)$ has pricing arbitrage since it is traded below the arbitrage free price $E$ 33.5. The extent for this pricing arbitrage for $\theta(A)=1$ is about $12 \%$.

Example 4.2 Estimate the stationary arbitrage free price of a movie pseudo asset $A$ with voice over dubbing and translations costing $E 40, E 3$, and $E 2$ respectively in a retail market operating from 13:00 Hrs to 15:00 Hrs if $p(A)$ follows the geometric Brownian motion.

Solution 4.2 Here, the business trading period $T=\frac{t}{24}=\frac{2}{24}$ since there 2 ${ }^{4} E$ stands for Emalangeni, an official currency of trade for the Kingdom of Eswatini (formerly Swaziland). The Emalangeni trades at par with the Rand $(R)$ of South Africa. 
hours between trading hours noon to 13 Hrs to 15 Hrs. Also, $X_{0}\left(A_{1}\right)=E 40$, $X_{0}\left(A_{2}\right)=E 3$ and $X_{0}\left(A_{3}\right)=E 2$. By [9],

$$
p=X(0, A) \mathrm{e}^{\left(\mu-\frac{1}{2} \sigma^{2}\right) t+\sigma t}
$$

In addition, $\mu=\frac{1}{2} \sigma^{2}$ is the optimal stationary point of $p$ as $t \rightarrow \infty$. Consequently,

$$
p=X(0, A) \mathrm{e}^{\sigma t}=X\left(0, A_{1}\right) \mathrm{e}^{\sigma t}+X\left(0, A_{2}\right) \mathrm{e}^{\sigma t}+X\left(0, A_{2}\right) \mathrm{e}^{\sigma t}
$$

Here, $\mu=\bar{X}(t, A)=E 15$ so that the standard deviation $\sigma=E 18$. Hence,

$$
p=E 40 \exp \left(\frac{36}{24}\right)+E 3 \exp \left(\frac{36}{24}\right)+E 2 \exp \left(\frac{36}{24}\right)
$$

Finally, one obtains that

$$
p=E 180+E 13.5+E 9=E 202.50
$$

Here, any $p(A)$ less than $E 202.50$ has arbitrage tendency.

From examples (4.1) and (4.2) above, it is clear that pseudo movie markets in the path of the geometric Brownian motion are more gaining to motion pictures owing to the magnitude of the finite variation process. Thus, the higher the volatility, the higher the dispersion of returns coupled with increased investment risk. These examples further demonstrate that pseudo movie markets may be closed or open depending on whether the market interacts with others through the free passage of information.

In this respect, closed pseudo movie markets exhibit more often the geometric pricing properties since there exists closed form pricing procedures in place visible in standard movie shops of mega investments and cinemas. The converse also holds good for open pseudo movie markets in pricing. This is in view of apparent flexibilities of open markets compared to closed markets generally as demonstrated in example (4.1). Essentially, example (4.1) in contrast with example (4.2) explains why open pseudo movie markets posses higher tendency for pricing arbitrage compared to closed pseudo movie markets where arbitrage tendencies might not even exists owing to the size of the price.

\section{Conclusion}

In this work, pricing movie industry pseudo assets and portfolio are studied. The work proved that pseudo assets trading in movie markets as add-ons present arbitrage opportunity capable of crunching movie markets. The work designed a stochastic pricing strategies under which such arbitrages can be eliminated. The process of eliminating pseudo asset arbitrage in movie pricing is demonstrated by means of examples for easy understanding. In conclusion, it is important to emphasize that for informed decisions on pseudo assets trading in movie markets, the knowledge of market type is significant. This provides some level of control over the hedging of portfolios to prevent pricing arbitrage and loss of investment resources. 


\section{Acknowledgements}

The authors acknowledge all the sources of literature used in this article, the anonymous referees and the editors of JMF involved in this work.

\section{Conflicts of Interest}

The authors declare no conflicts of interest regarding the publication of this article.

\section{References}

[1] Pangarker, N.A. and Smit, E.V.D.M. (2013) The Determinants of Box Office Performance in the Film Industry Revisited. South African Journal of Business Management, 44, 47-58. https://doi.org/10.4102/sajbm.v44i3.162

[2] Bhattacharjee, M. (2019) 3 Ways Bollywood Can Help Shape the Future of Globalization. World Economic Forum 2019.

https://www.weforum.org/agenda/2019/01/how-can-bollywood-shape-the-future-of -globalization/

[3] Orero, P. (2006) Synchronization in Voice-Over. In Bravo, J.M., Ed., A New Spectrum of Translation Studies, Publicationes de la Universidad de Valladolid, Valladolid, 225-264.

[4] Gemma, K. (2018) Contemporary French Cinema and the Langue de Passage: From Dheepan to Welcome. French Cultural Studies, 29, 39-48. https://doi.org/10.1177/0957155817738673

[5] Seong, W.P. (2009) The Present and Future of Americanization in South Korea. Journal of Futures Studies, 14, 51-66.

[6] Abed El-Rahman, T. (2015) Understanding Arab Culture through Cinema. Cultural Encounters, Conflicts, and Resolutions, 2, Article 7.

[7] Marshall, M. (1967). The Medium Is the Message. History of Communication Communications, Penguin Book Publication.

[8] Johan, S. and Lennart, L. (2019) Nonlinear System Identification: A User-Oriented Roadmap. 1-121. https://arxiv.org/pdf/1902.00683.pdf

[9] Oksendal, B. (2000) Stochastic Differential Equations. 5th Edition, Springer-Verlag, Berlin.

[10] Doshi, K. (2004) Arbitrage and International Trade and Finance. Qrius. https://qrius.com/arbitrage-and-international-trade-and-finance/

[11] Tuovila, A. (2019) Deadweight Loss. Investopedia. https://www.investopedia.com/terms/d/deadweightloss.asp

[12] Edmans A., Goldstein, I. and Jang, W. (2015) Feedback Effects, Asymmetric Trading, and the Limits to Arbitrage. American Economic Review, 105, 3766-3797. https://doi.org/10.1257/aer.20141271

[13] Munday, J. (2009) The Routledge Companion to Translation Studies. Routledge, Abingdon, 304.

[14] Mares, P. (2011) Media Consulting Group. Study on the Use of Subtitling. EACEA, Brussels.

[15] Sepielak, K. (2011) Voice-Over versus Voice-Over: Professional Point of View on Voice-Over in Poland. The 4th International Media for All Conference-Audivisual Translation: Taking Stock, London, 28 June-1 July 2011.

[16] Grigaraviciute, I. and Gottlieb, H. (1999) Danish Voices, Lithuanian Voice-Over: 
The Mechanics of Non-Synchronous Translation. Perspectives, 7, 41-80. https://doi.org/10.1080/0907676X.1999.9961347

[17] Wozniak, M. (2012) Voice-Over or Voice-in-Between? Some considerations about the Voice-Over Translation of Feature Films on Polish Television. In: Remael, A., Orero, P. and Carrol, M., Eds., Audiovisual Translation and Media Accessibility at the Crossroads, Media for All, Amsterdam, 209-228.

https://doi.org/10.1163/9789401207812_013

[18] Hennig-Thurrau, T., Houston, M.B. and Sridhar, S. (2005) Can Marketing Carry a Bad Product? Evidence from the Motion Picture Industry. Marketing Letters, 17, 205-219. https://doi.org/10.1007/s11002-006-7416-0

[19] Eliashberg, J., Jonker, J.J., Sawhney, M.S. and Weirenga, B. (2001) MOVIEMOD: An Implementable Decision-Support System for Prerelease Market Evaluation of Motion Pictures. Marketing Science, 19, 226-243.

https://doi.org/10.1287/mksc.19.3.226.11796

[20] Einav, L. (2007) Seasonality in the U.S Motion Picture Industry. RAND Journal of Economics, 38, 273-295. https://doi.org/10.1111/j.1756-2171.2007.tb00048.x

[21] Dana, Jr., J.D. and Spier, K.E. (2001) Revenue Sharing in the Video Rental Industry. Industrial Economics, 49, 223-245. https://doi.org/10.1111/1467-6451.00147

[22] Mortimer, J.H. (2006) Vertical Contracts in the Video Rental Industry. Working paper, Havard Department of Economics, Havard.

[23] Prasad, A., Mahajjan, V. and Bronnenberg, B.J. (2004) Product Entry Timing in Dual Distribution Channels: The Case of the Movie Industry. Review of Marketing Science, 2. https://doi.org/10.2202/1546-5616.1008

[24] Zufryden, F.S. (1996) Linking Advertising to Box Office Performance of New Film Releases: A Markting Planning Model. Journal of Advertising Research, July-August 29-41.

[25] Sawhney, M.S. and Eliasberg, J. (1996) A Parsimonious Model for Forecasting Gross Box-Office Revenues of Motion Pictures. Marketing Science, 15, 113-131. https://doi.org/10.1287/mksc.15.2.113

[26] Lilien, G.L. and Rangaswamy, A. (2005) Marketing Engineering. Trafford Publishing, Bloomington. https://doi.org/10.2307/3151926

[27] Krider, R.E. and Weinberg, C.B. (1998) Competitive Dynamics and the Introduction of New Products: The Motion Picture Timing Game. Journal of Marketing Research, 35, 1-15. https://doi.org/10.2307/3151926

[28] Stroock, D. W. (2008) Partial Differential Equation for Probabilists. Cambridge University Press, Cambridge.

[29] Black, F. and Scholes, M. (1973) The Pricing of Options and Corporate Liabilities. Journal of Political Economy, 81, 637-654. https://doi.org/10.1086/260062

[30] Swami, S., Eliashberg, J. and Weinberg, C.B. (1999) SilverScreen: A Modeling Approach to Movie Screens Management. Marketing Science, 18, 352-372.

https://doi.org/10.1287/mksc.18.3.352

[31] Jeuland, A. and Shugan, S. (1983) Managing Channel Profits. Marketing Science, 2, 239-272. https://doi.org/10.1287/mksc.2.3.239

[32] Weirenga, B., Van Bruggen, G.H. and Staelin, R. (1999) The Success of Marketing Management Support Systems. Marketing Science, 18, 196-207.

https://doi.org/10.1287/mksc.18.3.196

[33] Gerchak, Y., Cho, R.K. and Ray, S. (2006) Coordination of Quantity and Shelf-Retention Timing in the Video Movie Rental Industry. IIE Transactions, 38, 525-536.

https://doi.org/10.1080/07408170590961166 